\title{
ALOCAÇÃO DE PÓLOS EM SISTEMAS LINEARES INVARIANTES NO TEMPO UTILIZANDO REALIMENTAÇÃO DA DERIVADA DE ESTADOS E A EQUAÇÃO DE LYAPUNOV
}

\author{
José M. Araújo* \\ jomario@cefetba.br
}

\author{
Alexandre C. Castro* \\ castro@cefetba.br
}

\author{
Eduardo T. F. Santos* \\ eduardot@cefetba.br \\ *Instituto Federal da Bahia, Departamento de Tecnologia em Eletro-Eletrônica \\ Grupo de Pesquisa em Sinais e Sistemas \\ Rua Emídio dos Santos, S/N, Barbalho \\ CEP 40301-015 - Salvador BA
}

\section{RESUMO}

A realimentação da derivada de estados tem recebido grande atenção em tempos recentes, por ser uma alternativa à realimentação de estados e ser capaz de, exceto nos casos em que há pelo menos um pólo na origem, prover a conhecida alocação de pólos de um sistema linear invariante no tempo, que é uma das principais aplicações da realimentação de estados. Com base em procedimentos já consolidados para alocação de pólos por realimentação de estados utilizando a solução da equação de Lyapunov, são apresentados procedimentos análogos para realimentação da derivada de estados, e então apresentadas proposições análogas às aplicadas para realimentação de estados. Exemplos são então apresentados, comprovando a validade de tais procedimentos.

PALAVRAS-CHAVE: Alocação de pólos, sistemas lineares, realimentação da derivada de estados, equação de Lyapunov.

\section{ABSTRACT}

The state-derivative feedback has received great attention in recent times, for being an alternative to the state feedback and to be capable of, except in the cases where it has at least

Artigo submetido em 06/11/2006 (Id.: 00764)

Revisado em 19/03/2007, 10/04/2008, 03/03/2009

Aceito sob recomendação do Editor Associado Prof. Liu Hsu one pole in origin, to provide the known pole assignment of a linear time-invariant system, one of the main applications of the state feedback. On the basis of procedures already consolidated for pole assignment with state feedback using the solution of the Lyapunov type equation, analogous procedures are presented for state-derivative feedback, and then considered analogous propositions to the applicable ones for state feedback. Examples then are presented, proving the validity of such procedures.

KEYWORDS: Pole assignment, linear systems, statederivative feedback, Lyapunov equation.

\section{INTRODUÇÃO}

A realimentação derivativa de estados é uma técnica de controle moderna que vem recebendo crescente atenção em tempos recentes. Algumas aplicações bem sucedidas são regularização em sistemas lineares singulares (Garcia-Planas, 2003) e alocação de pólos (Abdelaziz e Valášek, 2004; Abdelaziz e Valášek, 2005; Teixeira et al, 2006; Assunção et al, 2007). Ela tem sido aplicada de forma bem sucedida como uma alternativa à realimentação de estados (Duan et al, 2005; Boukas and Habetler, 2004), pois em certas situações, a derivada é mais facilmente medida ou estimada. Por exemplo, a partir da velocidade, a determinação da acelera- 
ção é uma estimativa simples (derivada), que não apresenta maiores problemas de ordem experimental. Já a posição tem que ser estimada a partir de uma integral, que enfrenta problemas práticos de estabilidade devido ao chamado off-set nos sensores.

Chen (1999) apresenta um estudo pormenorizado para realimentação de estados, em sistemas de entrada simples e de entradas múltiplas, onde procedimentos e teoremas são apresentados para alocação de pólos através da realimentação de estados baseados na solução da equação de Lyapunov, com a única restrição de que o conjunto de pólos desejados não tenha nenhum pólo comum com o sistema em malha aberta (Gantmacher, 1990). A seguir serão apresentados procedimentos e proposições análogos quando a realimentação da derivada dos estados é aplicada, e alguns exemplos numéricos serão usados para ilustrar o mérito da proposta.

\section{PRELIMINARES}

Seja o sistema linear invariante no tempo, descrito no espaço de estados:

$$
\dot{x}(t)=A x(t)+B u(t)
$$

Em que $A \in \Re^{n \times n}, B \in \Re^{n \times m}$. Caso $m=1$, a descrição é dita com entrada simples, sendo de entrada múltipla caso $m$ $>$ 1. A realimentação da derivada dos estados é dada pela lei de controle:

$$
u(t)=-F \dot{x}(t)
$$

O sistema em malha fechada a partir desta lei de controle tem a seguinte descrição:

$$
\dot{x}(t)=(I+B F)^{-1} A x(t)
$$

Tendo sido assumida a não-singularidade de $I+B F$. Seja $H$ uma matriz não-singular, assintoticamente estável, ou seja, todos os autovalores não nulos. O problema tem a seguinte formulação: dado o sistema original descrito pela equação (1), encontrar uma matriz de ganhos $F$ para realimentação da derivada dos estados, tal que os autovalores da matriz $(I+B F)^{-1} A$ sejam os mesmos de $H$. É fácil observar que não há solução se $A$ for singular, o que é abordado numa das proposições da próxima seção. Nos procedimentos e proposições a seguir, baseados na solução de uma equação tipo Lyapunov, apresenta-se a solução para o problema e as condições para sua existência.

\section{SISTEMAS COM ENTRADA SIMPLES}

Para a solução do problema de alocação de pólos por realimentação da derivada dos estados num sistema em que $u(t)$ é uma função escalar, propõe-se o procedimento que se segue:

Procedimento 3.1: Dado um sistema na forma da equação(1), com $A$ não-singular e o par $(A, B)$ controlável, os passos seguintes resolvem o problema da alocação de pólos:

i Escolhe-se uma matriz $H$, não-singular, com os autovalores desejados, nenhum deles em comum com a matriz A;

ii Escolhe-se arbitrariamente $\bar{F} \in \Re^{1 \times n}$, respeitando-se que o $\operatorname{par}(H, \bar{F})$ seja observável;

iii Resolve-se a equação de Lyapunov

$$
A T-T H=B \bar{F} H
$$

iv Calcula-se $F=\bar{F} T^{-1}$, que assegura que os autovalores de $(I+B F)^{-1} A$ são os mesmos de $H$.

As proposições enunciadas na seqüência contemplam todos as características mencionadas no procedimento 3.1 e asseguram a sua eficácia.

Proposição 3.1: A matriz $T$, não-singular, calculada de acordo com o procedimento 1 , é capaz de alocar os pólos do sistema se e somente se a matriz $A$ é não-singular.

Demonstração: É fácil verificar através da equação(4) levemente modificada:

$$
A=(I+B F) T H T^{-1}
$$

Que se $A$ for não-singular, então $I+B F$ é não-singular. Por outro lado, se $A$ for singular, $I+B F$ é singular, e os pólos do sistema não podem ser alterados para os autovalores da matriz $H$, uma vez que o sistema não pode ser escrito como na equação(3).

Lema 3.1: Sejam $A$ e $H$ matrizes $n \times n$, com espectro de $H \quad\left(\bar{\lambda}_{i}, m_{i}\right)$ (autovalores e respectivas multiplicidades algébricas), $i=1,2, \ldots, l \leq n$ e $\Delta(\lambda)=\lambda^{n}+\alpha_{1} \lambda^{n-1}+\ldots+$ $\alpha_{n-1} \lambda+\alpha_{n}$ o polinômio característico de $A$; então $\Delta(H)$ tem espectro $\left(\Delta\left(\bar{\lambda}_{i}\right), m_{i}\right)$.

Demonstração: Uma matriz H tem sempre uma forma similar: 


$$
\bar{H}=P H P^{-1}=\left[\begin{array}{cccc}
\Lambda_{1} & 0 & \ldots & 0 \\
0 & \Lambda_{2} & \ddots & 0 \\
\vdots & \vdots & \ddots & \vdots \\
0 & 0 & \ldots & \Lambda_{k}
\end{array}\right]
$$

Em que $\Lambda_{i} m_{i} \times m_{i}, i=1,2 \ldots, l \leq n$ é formado por blocos de Jordan no autovalor $\lambda_{i}$, sendo no mínimo um e no máximo $m_{i}$ blocos de Jordan, a depender da multiplicidade geométrica de $\bar{\lambda}_{i}$. Considere-se agora que o polinômio característico de A seja avaliado em $\bar{H}$. Isto resulta em:

$\Delta(\bar{H})=P \Delta(H) P^{-1}\left[\begin{array}{cccc}\Delta\left(\Lambda_{1}\right) & 0 & \ldots & 0 \\ 0 & \Delta\left(\Lambda_{2}\right) & \ddots & 0 \\ \vdots & \vdots & \ddots & \vdots \\ 0 & 0 & \ldots & \Delta\left(\Lambda_{k}\right)\end{array}\right]$

Da teoria de funções de matrizes, é direto observar que $\Delta\left(\Lambda_{i}\right)$ é triangular, para todo $i$, e as entradas na diagonal principal valem $\Delta\left(\bar{\lambda}_{i}\right)$ (Chen, 1999). Assim, fica demonstrado que o espectro de $\Delta(H)$, o mesmo de sua similar $\Delta(\bar{H})$, é $\left(\Delta\left(\bar{\lambda}_{i}\right), m_{i}\right)$.

Proposição 3.2: Se $A$ e $H$ são matrizes sem autovalores em comum, então a única solução $T$ da equação de Lyapunov $A T-T H=B \bar{F} H$ é não-singular se e somente se $(A, B)$ é controlável, $(H, \bar{F})$ é observável e $H$ é não-singular.

Demonstração: Sem perda de generalidade, escolheremos $n$ = 4, como em Chen (1999), para demonstração. O polinômio característico de $A$ será:

$$
\Delta(\lambda)=\lambda^{4}+\alpha_{1} \lambda^{3}+\alpha_{2} \lambda^{2}+\alpha_{3} \lambda+\alpha_{4}
$$

Pelo teorema de Cayley-Hamilton, têm-se que:

$$
\Delta(A)=A^{4}+\alpha_{1} A^{3}+\alpha_{2} A^{2}+\alpha_{3} A+\alpha_{4} I \equiv \mathrm{O}
$$

Seja $\bar{\lambda}_{i}, i=1,2,3 \ldots$, os autovalores de $H$, com multiplicidades algébricas quaisquer. Como $A$ e $H$ não tem autovalores comuns, então nenhum autovalor de $H$ satisfaz a equação característica de $A$, e este fato assegura que:

$$
\Delta\left(\bar{\lambda}_{i}\right) \neq 0, \forall \bar{\lambda}_{i}
$$

Como $\Delta\left(\bar{\lambda}_{i}\right)$ é autovalor de:

$$
\Delta(H)=H^{4}+\alpha_{1} H^{3}+\alpha_{2} H^{2}+\alpha_{3} H+\alpha_{4} I
$$

de acordo com o lema 3.1, fica também assegurado que $\Delta(H)$ é não-singular. A seguir, vamos substituir $A T=$ $B \bar{F} H+T H$ em $A^{2} T-T H^{2}$. Isto resulta em:

$$
A^{2} T-T H^{2}=A(A T)-T H^{2}=A B \bar{F} H+B \bar{F} H^{2}
$$

A repetição deste procedimento até a quarta potência leva ao seguinte conjunto de equações:

$$
\begin{gathered}
I T-T I=\mathrm{O} \\
A T-T H=B \bar{F} H \\
A^{2} T-T H^{2}=A B \bar{F} H+B \bar{F} H^{2} \\
A^{3} T-T H^{3}=A^{2} B \bar{F} H+A B \bar{F} H^{2}+B \bar{F} H^{3} \\
A^{4} T-T H^{4}=A^{3} B \bar{F} H+A^{2} B \bar{F} H^{2}+A B \bar{F} H^{3}+B \bar{F} H^{4}
\end{gathered}
$$

Multiplicando as quatro primeiras equações, respectivamente, pelos coeficientes $\alpha_{4}, \alpha_{3}, \alpha_{2}$ e $\alpha_{1}$ e somando-se membro a membro, conclui-se que:

$$
\begin{aligned}
& \Delta(A) T-T \Delta(H)=-T \Delta(H)= \\
& =\left[\begin{array}{llll}
B & A B & A^{2} B & A^{3} B
\end{array}\right]\left[\begin{array}{cccc}
\alpha_{3} & \alpha_{2} & \alpha_{1} & 1 \\
\alpha_{2} & \alpha_{1} & 1 & 0 \\
\alpha_{1} & 1 & 0 & 0 \\
1 & 0 & 0 & 0
\end{array}\right]\left[\begin{array}{c}
\bar{F} \\
\bar{F} H \\
\bar{F} H^{2} \\
\bar{F} H^{3}
\end{array}\right] H
\end{aligned}
$$

Dado que $\Delta(H)$ é não-singular, $T$ será não-singular quando $(A, B)$ for controlável, $(H, \bar{F})$ for observável e $H$ for nãosingular. Por outro lado, se $(A, B)$ for não-controlável e/ou $(H, \bar{F})$ for não-observável e/ou $H$ for singular, $T$ será singular.

É importante notar que este resultado é absolutamente compatível com aqueles demonstrados em Abdelaziz e Valášek (2004) e em Teixeira et al (2006), ou seja, não é possível mover um único pólo simples na origem por realimentação da derivada dos estados, nem é possível alocar pólos na origem (o que é pouco comum em aplicações práticas). 


\subsection{Exemplos numéricos}

\subsubsection{Sistema de segunda ordem}

Seja o sistema

$$
\dot{x}(t)=\left[\begin{array}{ll}
0 & 1 \\
1 & 0
\end{array}\right] x(t)+\left[\begin{array}{l}
0 \\
1
\end{array}\right] u(t)
$$

O par $(A, B)$ é controlável. Os autovalores desejados em malha fechada são -2 e -3 . Escolhemos

$$
H=\left[\begin{array}{cc}
-2 & 0 \\
0 & -3
\end{array}\right] \text { e } \bar{F}=\left[\begin{array}{ll}
1 & 1
\end{array}\right]
$$

Com esta escolha, resolve-se a equação (4) para obter

$$
T=\left[\begin{array}{rr}
\frac{2}{3} & \frac{3}{8} \\
-\frac{4}{3} & -\frac{9}{8}
\end{array}\right] \text { e } F=\bar{F} T^{-1}=\left[\begin{array}{rr}
-\frac{5}{6} & -\frac{7}{6}
\end{array}\right]
$$

É fácil verificar que os autovalores de $(I+B F)^{-1} A$ são os desejados, ou seja, -2 e -3 .

\subsubsection{Alocação de múltiplos pólos}

Neste exemplo é mostrada uma alocação de múltiplos pólos, uma situação que gera problemas de ordem numérica em muitos métodos. Seja o sistema:

$$
\dot{x}(t)=\left[\begin{array}{ccc}
0 & 1 & 0 \\
0 & 0 & 1 \\
3 & 1 & -3
\end{array}\right] x(t)+\left[\begin{array}{l}
0 \\
0 \\
1
\end{array}\right] u(t)
$$

Os pólos em malha fechada desejados são $-2,-2 \mathrm{e}-2$. A aplicação do procedimento 3.1 a este problema é feita com

$H=\left[\begin{array}{ccc}-2 & 1 & 0 \\ 0 & -2 & 1 \\ 0 & 0 & -2\end{array}\right]$ e $\bar{F}=\left[\begin{array}{lll}1 & 0 & 0\end{array}\right]$

Obtém-se, de forma eficiente, as matrizes:

$$
\begin{aligned}
& T=\left[\begin{array}{ccc}
0,6667 & -0,1111 & 0,6296 \\
-1,3333 & 0,8889 & -1,3704 \\
2,6667 & -3,1111 & 3,6296
\end{array}\right] \\
& F=\left[\begin{array}{lll}
-3,5000 & -5,2500 & -1,3750
\end{array}\right]
\end{aligned}
$$

Estas garantem a alocação múltipla a partir da escolha de $H$.

\subsubsection{Sistema de levitação magnética}

Este exemplo é abordado por Kuo e Golnaragh (2002), e é visto na figura 1, cuja equação de estado da dinâmica linearizada do sistema de levitação magnética é dada por:

$\dot{x}(t)=\left[\begin{array}{ccc}0 & 1 & 0 \\ \frac{g}{x_{01}} & 0 & -2\left(\frac{g}{M x_{01}}\right)^{1 / 2} \\ 0 & 0 & -\frac{R}{L}\end{array}\right] x(t)+\left[\begin{array}{c}0 \\ 0 \\ \frac{1}{L}\end{array}\right] u(t)$

Em que $x_{01}$ é a posição de equilíbrio da esfera sustentada pelo levitador, $x_{1}=\Delta y, x_{2}=\Delta \dot{y}, x_{3}=\Delta i$ e $u=$ $\Delta e$, todos relativos ao ponto de equilíbrio. Vamos considerar os parâmetros do levitador com os seguintes valores: $\mathrm{g}=9,81 \mathrm{~m} / \mathrm{s}^{2}, M=0,01 \mathrm{~kg}, R=0,5 \Omega, L=0,01 H$ e $x_{01}^{\bar{y}} 0,1 \mathrm{~m}$. Os pólos do sistema em malha aberta são $-50,-9,9045$ e 9, 9045. Para determinar a matriz de ganho pela solução da equação (4), vamos considerar pólos e malha fechada $-51,-11$, e -8 . Assim, escolheremos

$$
H=\left[\begin{array}{ccc}
-51 & 0 & 0 \\
0 & -11 & 0 \\
0 & 0 & -8
\end{array}\right] \text { e } \bar{F}=\left[\begin{array}{l}
1 \\
1 \\
1
\end{array}\right]^{T}
$$

As matrizes $T$ e $F$ são mostradas a seguir:

$$
\begin{aligned}
& T=\left[\begin{array}{ccc}
403,6372 & -243,9816 & 110,6498 \\
-20585 & 2684 & -885,1988 \\
-5100 & 28 & 19
\end{array}\right] \mathrm{e} \\
& F=\left[\begin{array}{c}
0,0637 \\
0,0064 \\
-0,0209
\end{array}\right]
\end{aligned}
$$

Uma simulação deste sistema com um deslocamento inicial em relação ao ponto de equilíbrio de $5 \mathrm{~mm}$ é mostrada na figura 2.

\section{SISTEMAS COM ENTRADAS MÚLTI- PLAS}

Agora, seja o caso de entradas múltiplas $m$, ou seja, $u(t) \in$ $\Re^{n \times m}$. Seguindo o mesmo raciocínio do caso de entrada simples, é dado à seguir o procedimento para alocação de pólos do sistema:

Procedimento 4.1: Dado um sistema na forma da equação (1), com A não-singular, os passos seguintes resolvem o problema da alocação de pólos: 


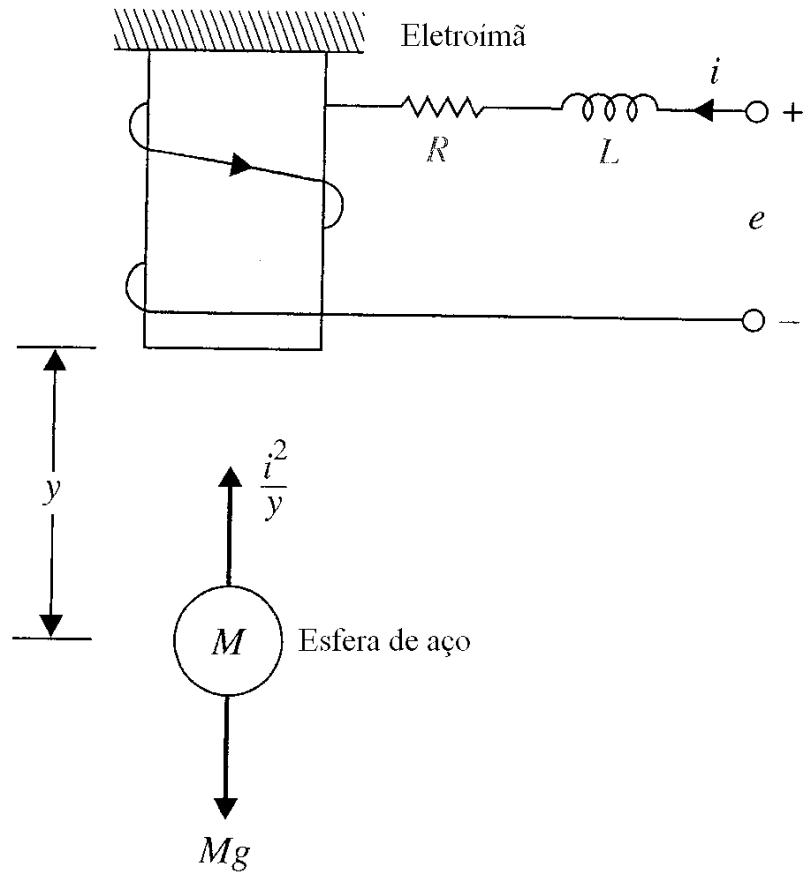

Figura 1: sistema de levitação magnética

i Escolhe-se uma matriz $H$, não-singular, com os autovalores desejados, nenhum deles em comum com a matriz A;

ii Escolhe-se arbitrariamente $\bar{F} \in \Re^{m \times n}$, respeitando-se

que o $\operatorname{par}(H, \bar{F})$ seja observável;

iii Resolve-se a equação de Lyapunov

$$
A T-T H=B \bar{F} H
$$

iv Caso $T$ seja singular, retorna-se aos passos ii e iii.

v Calcula-se $F=\bar{F} T^{-1}$, que assegura que os autovalores de $(I+B F)^{-1} A$ são os mesmos de $H$.

Nota-se que há uma diferença em relação ao caso com entrada simples. Assim enuncia-se a seguinte proposição para esta situação.

Proposição 4.1: Se $A$ e $H$ são matrizes sem autovalores em comum, então a única solução $T$ da equação de Lyapunov $A T-T H=B \bar{F} H$ é não-singular somente se $(A, B)$ é controlável, $(H, \bar{F})$ é observável e $H$ é não-singular.

Demonstração: sem perda de generalidade, pode-se escolher $n=4$, como em Chen (1999), para demonstração. Procedendo de forma semelhante àquela da proposição 3.2, encontra-se a seguinte identidade:

$$
\begin{aligned}
& \Delta(A) T-T \Delta(H)=-T \Delta(H)= \\
& =\left[\begin{array}{llll}
B & A B & A^{2} B & A^{3} B
\end{array}\right]\left[\begin{array}{cccc}
\alpha_{3} I & \alpha_{2} I & \alpha_{1} I & I \\
\alpha_{2} I & \alpha_{1} I & I & 0 \\
\alpha_{1} I & I & 0 & 0 \\
I & 0 & 0 & 0
\end{array}\right]\left[\begin{array}{c}
\bar{F} \\
\bar{F} H \\
\bar{F} H^{2} \\
\bar{F} H^{3}
\end{array}\right] H= \\
& =I J \Sigma V H
\end{aligned}
$$

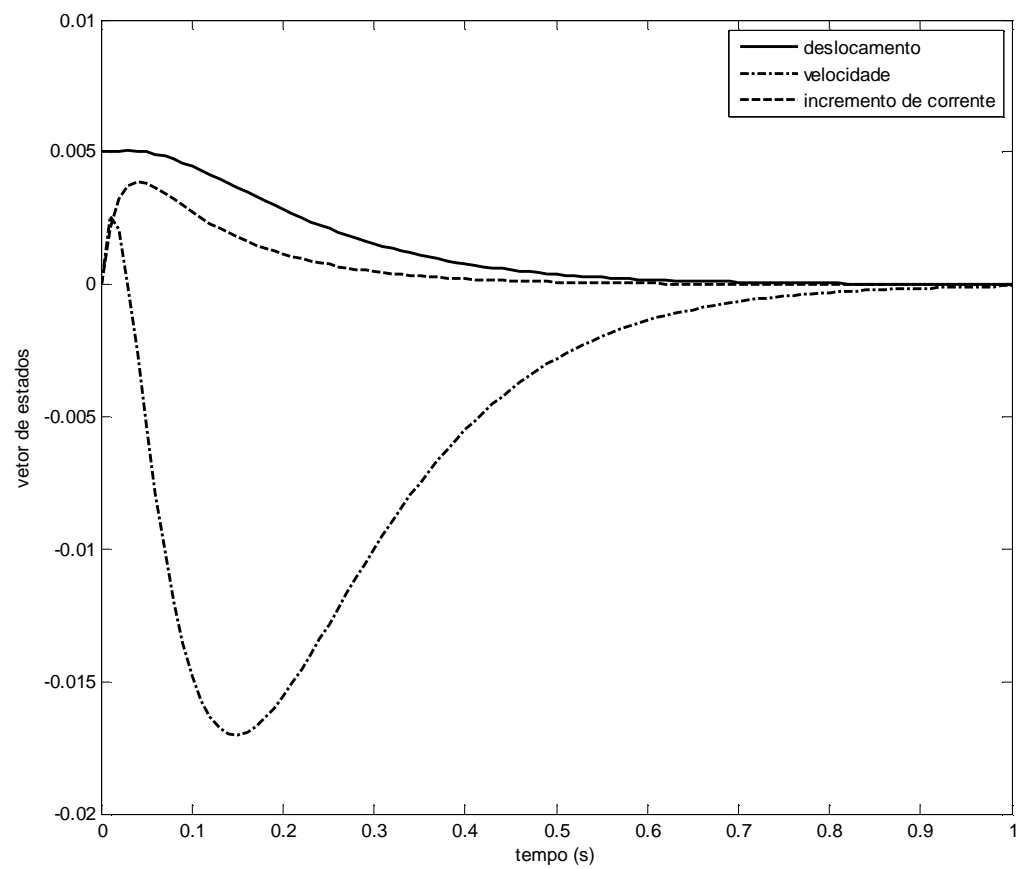

Figura 2: Resposta livre do sistema de levitação magnética com realimentação da derivada dos estados. 
As dimensões de $U, \Sigma, V$ e $H$ são respectivamente $n \times n m$, $n m \times n m, n m \times n$ e $n \times n$. Se $U$ ou $V$ não tiverem posto pleno, ou seja, (A,B) não controlável ou $(H, \bar{F})$ não observável, ou ainda $H$ for singular, $T$ será singular, pela nãosingularidade de $\Delta(H)$. No entanto, o fato de $(A, B)$ ser controlável, $(H, \bar{F})$ ser observável e $H$ ser não-singular não implica na não-singularidade de $T$. Logo, as condições da proposição são necessárias, porém, não são suficientes.

A proposição 3.1, demonstrada anteriormente, continua sendo aplicável no caso de sistema com múltiplas entradas.

\subsection{Exemplos numéricos}

\subsubsection{Sistema mecânico}

Este exemplo é apresentado por Teixeira et al (2006). A figura 3 apresenta o sistema em estudo. As equações de estado para a situação mostrada são:

$\dot{x}(t)=\left[\begin{array}{cccc}0 & 1 & 0 & 0 \\ \frac{-k_{1}}{m_{1}} & \frac{-b_{1}}{m_{1}} & 0 & \frac{b_{1}}{m_{1}} \\ 0 & 0 & 0 & 1 \\ 0 & \frac{b_{1}}{m_{2}} & \frac{-k_{2}}{m_{2}} & \frac{-b_{1}}{m_{2}}\end{array}\right] x(t)+\left[\begin{array}{cc}0 & 0 \\ \frac{1}{m_{1}} & 0 \\ 0 & 0 \\ 0 & \frac{1}{m_{2}}\end{array}\right] u(t)$

Em que $x_{2}=\dot{x_{1}} \mathrm{e} x_{4}=\dot{x_{3}}$. Os parâmetros utilizados foram $m_{1}=10 \mathrm{~kg}, m_{2}=30 \mathrm{~kg}, k_{1}=2,5 \mathrm{kN} / \mathrm{m}, k_{2}=1,5 \mathrm{kN} / \mathrm{m}$ e $b_{1}=$ $30 N s / m$. Deseja-se alocar os pólos do sistema nas posições: $-10,-15$ e $-2 \pm 10 j$. Escolhemos $H$ na forma canônica modal e $\bar{F}$ de acordo com o procedimento 4.1 :

$$
H=\left[\begin{array}{cccc}
-10 & 0 & 0 & 0 \\
0 & -15 & 0 & 0 \\
0 & 0 & -2 & 10 \\
0 & 0 & -10 & -2
\end{array}\right] \text { e } \bar{F}=\left[\begin{array}{ll}
1 & 0 \\
1 & 0 \\
1 & 1 \\
1 & 0
\end{array}\right]^{T}
$$

Esta escolha levada à equação (4) resulta em $T$ e $F$ mostrados à seguir:

$$
\begin{aligned}
T & =\left[\begin{array}{cccc}
0,0031 & 0,0035 & 0,0075 & -0,0049 \\
-0,0315 & -0,0526 & 0,0343 & 0,0845 \\
-0,0002 & -0,0002 & 0,0008 & 0,0047 \\
0,0022 & 0,0030 & -0,0484 & -0,0012
\end{array}\right] \\
F & =\left[\begin{array}{cccc}
407,9668 & 9,6443 & 483,9623 & 57,2625 \\
9,4479 & -0,5437 & 14,9122 & -19,3481
\end{array}\right]
\end{aligned}
$$

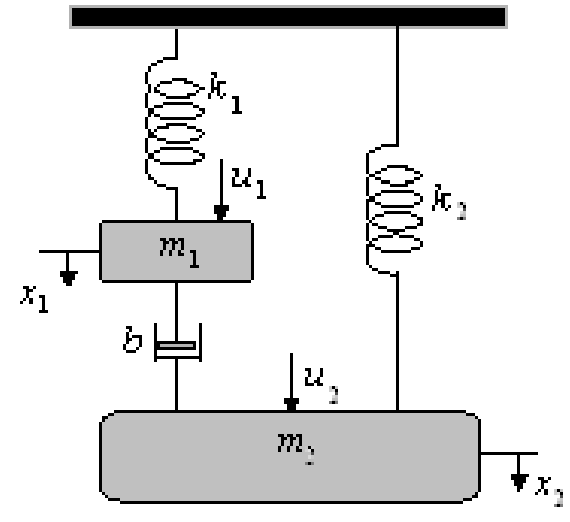

Figura 3: Sistema mecânico do exemplo 4.1.1.

Pode-se verificar que os autovalores de $(I+B F)^{-1} A$ são aqueles desejados no exemplo. A figura 4 mostra uma simulação do sistema em malha fechada, com o ganho calculado, para uma condição inicial $x^{T}(0)=\left[\begin{array}{llll}0,1 & 0 & 0,1 & 0\end{array}\right]$.

\subsubsection{Duas escolhas diferentes de $\bar{F}$}

Este exemplo é visto em Chen (1999), e será explorado o efeito de diferentes escolhas da matriz $\bar{F}$. O sistema é descrito por

$$
\dot{x}(t)=\left[\begin{array}{cccc}
0 & 1 & 0 & 0 \\
0 & 0 & 1 & 0 \\
-3 & 1 & 2 & 3 \\
2 & 1 & 0 & 0
\end{array}\right] x(t)+\left[\begin{array}{cc}
0 & 0 \\
0 & 0 \\
1 & 2 \\
0 & 2
\end{array}\right] u(t)
$$

As condições das proposições 3.1 e 4.1 são satisfeitas para $A$ e para o par $(A, B)$, respectivamente. Deseja-se alocar os pólos em malha fechada nas posições $-4 \pm 3 j \mathrm{e}-5 \pm 4 j$. Far-se-á duas escolhas de $\bar{F}$, e $H$ na forma modal:

$$
\begin{aligned}
& H=\left[\begin{array}{cccc}
-4 & 3 & 0 & 0 \\
-3 & -4 & 0 & 0 \\
0 & 0 & -5 & 4 \\
0 & 0 & -4 & -5
\end{array}\right], \\
& \bar{F}_{1}=\left[\begin{array}{ll}
1 & 0 \\
0 & 0 \\
1 & 0 \\
0 & 0
\end{array}\right]^{T} \text { e } \bar{F}_{2}=\left[\begin{array}{ll}
1 & 0 \\
0 & 0 \\
0 & 1 \\
0 & 0
\end{array}\right]^{T}
\end{aligned}
$$

Para o par $\left(H, \bar{F}_{1}\right)$, obtém-se $T$ e $F_{1}$ dados por: 


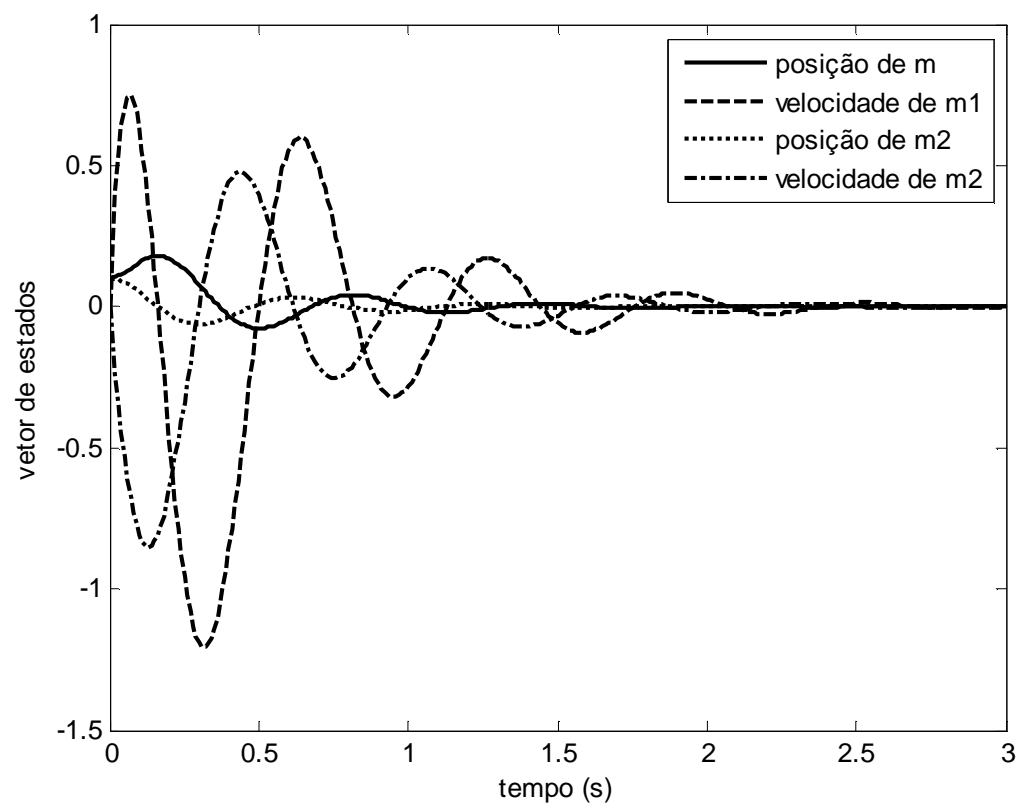

Figura 4: Resposta livre do sistema mecânico com realimentação da derivada de estados.

$$
\begin{aligned}
& T=\left[\begin{array}{cccc}
-0,0126 & -0,0273 & -0,0068 & -0,0182 \\
0,1322 & 0,0714 & 0,1070 & 0,0639 \\
-0,7428 & 0,1108 & -0,7905 & 0,1081 \\
-0,0151 & -0,0155 & -0,0087 & -0,0125
\end{array}\right] \\
& F_{1}=\left[\begin{array}{cccc}
1,8371 & 1,8946 & -1,0059 & -1,6917 \\
0 & 0 & 0 & 0
\end{array}\right]
\end{aligned}
$$

$F_{1}$ modifica os pólos do sistema para a posição desejada. Utiliza-se agora o $\operatorname{par}\left(H, \bar{F}_{2}\right)$, obtendo-se $T$ e $F_{2}$ :

$$
\begin{aligned}
& T=\left[\begin{array}{cccc}
-0,0126 & -0,0273 & -0,0193 & -0,0191 \\
0,1322 & 0,0714 & 0,1731 & 0,0185 \\
-0,7428 & 0,1108 & -0,9393 & 0,5998 \\
-0,0151 & -0,0155 & -2,0183 & -0,0107
\end{array}\right] \\
& F_{2}=\left[\begin{array}{cccc}
55,8587 & 19,6281 & 1,1880 & 0,5960 \\
0,0716 & -0,0748 & -0,0043 & -0,5005
\end{array}\right]
\end{aligned}
$$

Esta segunda matriz também move os pólos do sistema para a posição desejada, e a conclusão é que a solução não é única no caso de múltiplas entradas.

\section{CONCLUSÃO}

Foram propostos procedimentos e demonstradas proposições, balizados naqueles já existentes para realimentação de estados, de forma a alocar pólos de um sistema linear a partir da solução de uma equação tipo Lyapunov resultante da realimentação da derivada dos estados. Tais procedimentos foram testados em exemplos numéricos variados, em sistemas com entrada simples e entradas múltiplas, e sua efetividade foi confirmada, até mesmo em caso de alocação de pólos com multiplicidade maior que um. Nota-se que a única diferença em relação aos procedimentos usados em realimentação de estados é o requerimento que $A$ e $H$ sejam não-singulares, um resultado já confirmado para este tipo de técnica de realimentação utilizando outras metodologias. Também, no caso de múltiplas entradas, verificou-se que a matriz de realimentação da derivada de estados que soluciona o problema de alocação de pólos não é única.

\section{AGRADECIMENTOS}

Os autores gostariam de agradecer ao Instituto Federal da Bahia, pelo incentivo dado à condução de pesquisas na área de sinais e sistemas, e aos revisores anônimos, que muito contribuiram para melhorar a qualidade do presente trabalho.

\section{REFERÊNCIAS}

Abdelaziz, T.H.S. and Valášek, M. (2004). Pole placement for SISO linear systems by state-derivative feedback. IEE Proc.-Control Theory Appl., vol. 151, n. 4.

(2005). Direct algorithm for pole placement by state-derivative feedback for multi-input linear systems 
- Nonsingular case. Kybernetika, vol. 41, n. 5, pp. 637660.

Assunção, E., Teixeira ,M.C.M., Faria, F.A., Da Silva, N.A.P. and Cardim, R. (2007). Robust state-derivative feedback LMI-based designs for multivariable linear systems. International Journal of Control, vol. 80, n. 8, pp. 1260-1270.

Boukas, T.K. and Habetler, T.G. (2004). High-performance induction motor speed control using exact feedback linearization with state and state derivative feedback. IEEE Transactions on Power Electronics, vol. 19, n. 4, pp. $1022-1028$.

Chen, C. T. (1999). Linear System theory and design. Oxford University Press, New York.

Duan, Y. F., Ni , Y. Q. and Ko, J. M. (2005). State-Derivative feedback control of cable vibration using semiactive magnetorheological dampers. Computer-Aided Civil and Infrastructure Engineering, vol. 20, pp 431 - 449.

Gantmacher, F. R. (1990). Matrix Theory, vol. 01. American Mathematical Society, Providence.

Garcia-Planas, M. I. (2003). Regularizing Generalized Linear Systems by means a Derivative Feedback. Proceedings of international Conference on Physics and Control, Saint Petersburg, Russia, pp. 1134-1140.

Kuo, B. C. and Golnaraghi, F (2002). Automatic control systems. Wiley, New Jersey.

Teixeira, M. C. M.; Assunção, E.; Cardim, R e Covacic, M. R. (2006). Realimentação da derivada de estados a partir do projeto com realimentação de estados. Anais do XVI Congresso Brasileiro de Automática. Salvador, Bahia, Brasil. pp. 726-731. 